\title{
Universal Approach to Cosmological Singularities in Two Dimensional Dilaton Gravity
}

\author{
Gabriel N. Martin ${ }^{1}$ and Francisco D. Mazzitelli ${ }^{1,2}$ \\ ${ }^{1}$ Departamento de Física, Facultad de Ciencias Exactas y Naturales \\ Universidad de Buenos Aires- Ciudad Universitaria, Pabellón I \\ 1428 Buenos Aires, Argentina \\ ${ }^{2}$ Instituto de Astronomía y Física del Espacio \\ Casilla de Correo 67-Sucursal 28 \\ 1428 Buenos Aires, Argentina
}

\begin{abstract}
We show that in a large class of two dimensional models with conformal matter fields, the semiclassical cosmological solutions have a weak coupling singularity if the classical matter content is below a certain threshold. This threshold and the approach to the singularity are model-independent. When the matter fields are not conformally invariant, the singularity persists if the quantum state is the vacuum near the singularity, and could dissappear for other quantum states.
\end{abstract}

April 1994 


\section{INTRODUCTION}

In the last two years there has been a lot of activity in the study of $(1+1)$-dimensional models of gravity. The models include the spacetime metric, a dilaton and $N$ conformal matter fields, and are useful toy models to address the problem of black hole formation and evaporation, including backreaction effects.

The original theory proposed by Callan, Giddings, Harvey and Strominger (CGHS) is [1]

$$
\begin{aligned}
S_{C G H S} & =\frac{1}{2 \pi} \int d^{2} x \sqrt{-g}\left[e^{-2 \phi}\left(R+4(\nabla \phi)^{2}+4 \lambda^{2}\right)-\frac{1}{2} \sum_{i=1}^{N}\left(\nabla f_{i}\right)^{2}\right] \\
& -\frac{\kappa}{8 \pi} \int d^{2} x \sqrt{-g} R \frac{1}{\nabla^{2}} R
\end{aligned}
$$

where $\phi$ is the dilaton, $f_{i}$ are the matter fields and $\kappa=N / 12$. The term proportional to $\kappa$ is the well known conformal anomaly, and comes from one-loop quantum corrections. The model is exactly soluble at the classical level $(\kappa=0)$. However, the semiclassical CGHS equations have not been analytically solved.

It is possible to modify the gravity-dilaton couplings of the theory in order to find exactly soluble semiclassical equations [2]- [3]. In particular, in Ref. [3], Russo, Susskind and Thorlacius (RST) obtained a soluble model imposing the classical conservation law

$$
\partial_{\mu} \partial^{\mu}(\rho-\phi)=0
$$

to be preserved at the semiclassical level. This leads to the RST semiclassical action

$$
S_{R S T}=S_{C G H S}-\frac{\kappa}{4 \pi} \int d^{2} x \sqrt{-g} \phi R
$$

For $\kappa=(N-24) / 12$ Eq.3 describes a conformal field theory with vanishing total central charge.

The RST model is not only useful for the analysis of black hole evaporation. It has also been used to study the effect of backreaction on the classical cosmological solutions. In par-

ticular, it has been shown that, if the classical matter content is below certain threshold, the 
semiclassical cosmological solutions develop a weak coupling singularity [4]. This result is in contradiction with the standard lore: quantum effects do not help to smear the cosmological singularities but generate new singularities at weak coupling. ${ }^{1}$

Since the RST model contains an additional symmetry that leads to the conservation law Eq. A, one may suspect that some of the results may not be generic, and that other models like CGHS may give qualitatively different results [ [6]. In this work we will analyze the issue of the weak coupling cosmological singularities in a model with arbitrary graviton-dilaton couplings

$$
\begin{aligned}
S & =\frac{1}{2 \pi} \int d^{2} x \sqrt{-g}\left[A(\phi)\left(R+4(\nabla \phi)^{2}+4 \lambda^{2}(\phi)\right)-\frac{1}{2} \sum_{i=1}^{N}\left(\nabla f_{i}\right)^{2}\right] \\
& -\frac{\kappa}{8 \pi} \int d^{2} x \sqrt{-g} R \frac{1}{\nabla^{2}} R
\end{aligned}
$$

We will see that, for a large class of couplings $A(\phi)$ and $\lambda(\phi)$, the weak coupling singularity is present. Moreover, we will prove that the threshold and the leading behaviour of the scale factor near the singularity are independent of the couplings. Finally, we will consider non-conformally invariant matter fields. We will prove that for a particular choice of the quantum state of the matter fields the singularity does not dissappear. However, we will also argue that, for other quantum states, cosmological particle creation may wash the singularity away.

\section{COSMOLOGICAL SINGULARITIES}

In the conformal gauge $g_{++}=g_{--}=0, g_{+-}=-\frac{1}{2} e^{2 \rho}$, the equations of motion derived from Eq.⿴囗十

$$
\begin{aligned}
& -A^{\prime} \partial_{+} \partial_{-} \rho-4 A \partial_{+} \partial_{-} \phi-2 A^{\prime} \partial_{-} \phi \partial_{+} \phi-e^{2 \rho}\left(\lambda \lambda^{\prime} A+\frac{1}{2} \lambda^{2} A^{\prime}\right)=0 \\
& A^{\prime \prime} \partial_{+} \phi \partial_{-} \phi+\kappa \partial_{+} \partial_{-} \rho+A^{\prime} \partial_{+} \partial_{-} \phi+\lambda^{2} A e^{2 \rho}=0
\end{aligned}
$$

\footnotetext{
${ }^{1} \mathrm{~A}$ result of this type has also been found in Ref. [5] for $N=24$ in a different model.
} 


$$
\begin{aligned}
& \left(A^{\prime \prime}-4 A\right)\left(\partial_{ \pm} \phi\right)^{2}+A^{\prime} \partial_{ \pm} \partial_{ \pm} \phi-2 A^{\prime} \partial_{ \pm} \phi \partial_{ \pm} \rho+\frac{1}{2} \sum_{i=1}^{N}\left(\partial_{ \pm} f_{i}\right)^{2} \\
& -\kappa\left[\left(\partial_{ \pm} \rho\right)^{2}-\partial_{ \pm} \partial_{ \pm} \rho\right]=\kappa t_{ \pm}
\end{aligned}
$$

where the prime denotes a derivative with respect to $\phi$. The functions $t_{ \pm}\left(x^{ \pm}\right)$depend on the quantum state of the matter fields and come from the variation of the anomaly term.

The solutions to the above equations can be trusted only in weak coupling regions where the quantum corrections to the semiclassical action Eq.14 are small. These quantum corrections will be supressed by a $\phi$-dependent coupling given by $g_{c}^{2} \sim \frac{1}{A^{2}}$. If the quantum corrections are calculated with the action Eq. 4 that includes the trace anomaly, they will be supressed by an effective coupling $g_{\text {eff }}$ which is given by the inverse of the determinant of the $\rho-\phi$ target space metric in Eq.4 (see Refs. [7], [4]). For our model we obtain

$$
g_{\text {eff }}^{2}=\frac{1}{\left|A^{\prime 2}-4 \kappa A\right|}
$$

so the weak coupling region is defined by $g_{e f f} \ll 1$ or $g_{c} \ll 1$. For simplicitly in what follows we will assume that $A>0$ and $A^{2} \gg \kappa A$ in the weak coupling region.

The equations of motion can be written in a simpler form in terms of the new fields $X$ and $Y$ defined as

$$
\begin{aligned}
& X=\kappa \rho+A(\phi) \\
& Y=\int d \phi \sqrt{\left|\frac{1}{4 \kappa} A^{2}-A\right|}
\end{aligned}
$$

Indeed, the equations of motion read

$$
\begin{aligned}
\partial_{+} \partial_{-} X & =-\lambda^{2} A e^{2 \rho} \\
\partial_{+} \partial_{-} Y & =\frac{1}{8 Y^{\prime}} e^{2 \rho}\left[A^{\prime} \lambda^{2}+2 \lambda \lambda^{\prime} A-\frac{2}{\kappa} A A^{\prime} \lambda^{2}\right] \\
\kappa t_{ \pm} & =\frac{1}{2} \sum_{i=1}^{N} \partial_{ \pm} f_{i} \partial_{ \pm} f_{i}+4 \partial_{ \pm} Y \partial_{ \pm} Y-\frac{1}{\kappa} \partial_{ \pm} X \partial_{ \pm} X+\partial_{ \pm}^{2} X
\end{aligned}
$$

For $\lambda(\phi)=0, X$ and $Y$ are free fields and the equations can be trivially solved. This fact will be important in what follows. 
Let us now consider time-dependent cosmological solutions. In coordinates $\sigma^{ \pm}=\tau \pm \sigma$ the two dimensional metric is given by

$$
d s^{2}=-e^{2 \rho(\tau)} d \sigma^{+} d \sigma^{-}
$$

To solve the equations of motion we must fix the functions $t_{ \pm}$. Since the quantum matter fields are conformally invariant, a natural choice for the quantum state is the conformal vacuum [8], in which $t_{ \pm}\left(\sigma^{ \pm}\right)=0$. With this choice, the solutions for $\lambda=0$ are

$$
\begin{aligned}
X & =k_{1} \tau+\text { const } \\
Y & =k_{2} \tau+\text { const } \\
0 & =\frac{1}{8} \sum_{i=1}^{N} \dot{f}_{i}^{2}-\frac{1}{4 \kappa} k_{1}^{2}+k_{2}^{2}
\end{aligned}
$$

where $k_{1}$ and $k_{2}$ are integration constants. If $\sum_{i=1}^{N} \dot{f}_{i}^{2} \neq 0, k_{1}$ cannot vanish and without loss of generality we can choose the coordinate $\tau$ such that $k_{1}=\frac{\kappa}{T}$, where $T$ is an arbitrary time scale.

From the above solution we observe that the classical matter content defined as $m^{2}=$ $\frac{1}{8} \sum_{i=1}^{N} \dot{f}_{i}^{2} T^{2}$ must satisfy $0 \leq m^{2} \leq m_{c r}^{2}=\frac{\kappa}{4}$. If $m^{2}$ exceeds the critical value $m_{c r}^{2}$, there is no solution. When $m^{2}$ equals the critical value, $\phi$ is constant and $\rho$ is a linear function of $\tau$. The spacetime metric describes a two dimensional Milne universe [9]. The scalar curvature $R=2 \ddot{\rho} e^{-2 \rho}$ vanishes. This is to be expected, since the Milne universe is merely a non trivial coordinatization of flat spacetime. This result is independent of the function $A(\phi)$.

We will now show that, if $m^{2}<m_{c r}^{2}$, the semiclassical solutions develop a weak coupling singularity for $\tau \rightarrow-\infty$. Let us denote by $\delta$ the distance between the actual matter content and the threshold, i.e., $\delta \equiv \sqrt{m_{c r}^{2}-m^{2}}$. We obtain

$$
\begin{aligned}
& X=A(\phi)+\kappa \rho=\frac{\kappa \tau}{T}+\text { const } \\
& Y=\int d \phi \sqrt{\frac{1}{4 \kappa} A^{\prime 2}-A}=\frac{\delta|\tau|}{T}+\text { const }
\end{aligned}
$$

In the weak coupling region we have $A^{\prime 2} \gg \kappa A$. Therefore, 


$$
A-2 \kappa \int d \phi \frac{A}{A^{\prime}} \approx 2 \sqrt{\kappa} \delta \frac{|\tau|}{T}+\text { const }
$$

From this equation we see that in general $A(\tau)$ is not a linear function 1 of $\tau$. Consequently, $\ddot{\rho}=-\frac{1}{\kappa} \ddot{A}$ is different from zero and the scalar curvature $R$ diverges for $\tau \rightarrow-\infty$.

It is instructive to see the solutions in some particular cases. The condition $A^{\prime 2} \gg \kappa A$ is satisfied, for example, for couplings of the form $A(\phi)=e^{-\gamma \phi}$, for $\phi \rightarrow-\infty$. In this case

$$
\begin{aligned}
& \phi(\tau) \approx-\frac{1}{\gamma} \ln \left[2 \sqrt{\kappa} \delta \frac{|\tau|}{T}\right] \rightarrow-\infty(\tau \rightarrow-\infty) \\
& A(\tau) \approx 2 \sqrt{\kappa} \delta \frac{|\tau|}{T}+\frac{2 \kappa}{\gamma^{2}} \ln \left[2 \sqrt{\kappa} \delta \frac{|\tau|}{T}\right] \\
& \rho(\tau) \approx\left(1+\frac{2 \delta}{\sqrt{\kappa}}\right) \frac{\tau}{T}-\frac{2}{\gamma^{2}} \ln \left[2 \sqrt{\kappa} \delta \frac{|\tau|}{T}\right] \\
& R(\tau) \approx \frac{4}{\gamma^{2} \tau^{2}} e^{-2 \rho} \rightarrow \infty(\tau \rightarrow-\infty)
\end{aligned}
$$

Similar results can be obtained for other families of couplings. For example, for $A(\phi)=$ $\phi^{2 n}, n>1$ we have

$$
\begin{aligned}
& \phi(\tau) \approx\left(2 \sqrt{\kappa} \delta \frac{|\tau|}{T}\right)^{\frac{1}{2 n}} \\
& A(\tau) \approx 2 \sqrt{\kappa} \delta \frac{|\tau|}{T}+\frac{\kappa}{n}\left(2 \sqrt{\kappa} \delta \frac{|\tau|}{T}\right)^{\frac{1}{n}} \\
& \rho(\tau) \approx\left(1+\frac{2 \delta}{\sqrt{\kappa}}\right) \frac{\tau}{T}-\frac{1}{n}\left(2 \sqrt{\kappa} \delta \frac{|\tau|}{T}\right)^{\frac{1}{n}} \\
& R(\tau) \approx \frac{2(n-1)}{n^{3}}(2 \sqrt{\kappa} \delta|\tau|)^{\frac{1}{n}} \frac{1}{\tau^{2}} e^{-2 \rho} \rightarrow \infty(\tau \rightarrow-\infty)
\end{aligned}
$$

The general result is that, as long as $A^{\prime 2} \gg \kappa A$, a weak coupling singularity takes place. The threshold and the leading behaviour of the Liouville field $\rho(\tau)$ are independent of the function $A(\phi)$. Other quantities like the subleading correction to $\rho(\tau)$ and the scalar curvature do depend on this function.

\footnotetext{
${ }^{2}$ In the particular case $A(\phi)$ proportional to $\phi^{2}, Y$ is proportional to $A$. Therefore $A$ is linear in $\tau$ and we have a Milne universe for any matter content. However, this particular $A(\phi)$ does not satisfy our hypothesis $A^{\prime 2} \gg \kappa A$
} 
Up to here we assumed that the 'potential' $\lambda^{2}$ vanishes. It is easy to see that the results are valid for a large class of potentials. Indeed, as long as

$$
R \gg \max \left\{\lambda^{2} A, \quad\left|\left(\lambda^{2}+2 \frac{A}{\dot{A}} \lambda \dot{\lambda}\right)\right|\right\}
$$

the terms containing $\lambda^{2}$ can be neglected in the equations of motion. As a consequence, the analysis of the existence of the singularities needs no modifications. It is worth remarking that the condition Eq.17 is not too restrictive: the scalar curvature diverges like $e^{2\left(1+\frac{2 \delta}{\sqrt{\kappa}}\right)|\tau|}$ times a power of $\tau$ while $A(\tau)$ diverges linearly in $\tau$. Therefore, the above conditions are satisfied unless $\lambda(\phi)$ has a strong divergence in the weak coupling region.

Finally we point out that, when $\lambda^{2} \neq 0$, we cannot conclude from our calculations that there is no solution when the matter content is above the threshold. Indeed, in the RST model there is a solution for any matter content [4]. Of course, the solution does not have weak coupling singularities when $m^{2} \geq m_{c r}^{2}$, in agreement with the analysis presented here.

The existence of the threshold and a similar dependence with the logarithm of $\delta$ (see Eq.15) have also been found in the analysis of gravitational collapse within the RST model [10]. Therefore our analysis suggests that, also in that problem, the same threshold should

appear for other theories. However, the presence of $\log [\delta]$ in the expression for the mass of the black hole would be particular of the exponential couplings.

\section{NON MINIMAL COUPLING: BREAKING OF CONFORMAL INVARIANCE}

Let us now consider the case of non-conformally coupled matter fields. We add to the classical action the term

$$
\Delta S=\frac{\xi}{4 \pi} \int d^{2} x \sqrt{-g} R \sum_{i=1}^{N} f_{i}^{2}
$$

where $\xi$ is an arbitrary constant. In principle, one can compute the effective action and the effective semiclassical equations induced by the non conformal matter fields using an expansion in powers of $\xi$. However, these equations are non-local (even in the conformal 
gauge) and extremely difficult to solve. We will follow here a simpler and more qualitative approach. It is well known that breaking of conformal invariance induces particle creation. The energy density of the created particles (which we will denote by $\epsilon$ ) contributes as a classical source, i.e. with a positive sign, in Eq. 12. Therefore, if $\epsilon \sim \frac{m_{c r}^{2}}{T^{2}}$, then the matter content would be greater than the threshold and the singularity could dissapear.

Any of the scalar fields $f_{i}$ can be expanded as

$$
f(\tau, x)=\int \frac{d k}{2 \pi} e^{i k x}\left[a_{k} f_{k}(\tau)+a_{-k}^{\dagger} f_{k}^{*}(\tau)\right]
$$

where $a_{k}$ and $a_{k}^{\dagger}$ are the usual creation and annihilaton operators. The modes $f_{k}$ satisfy

$$
\ddot{f}_{k}+\left[k^{2}+\xi R e^{2 \rho}\right] f_{k}=0
$$

In terms of the functions $\alpha(\tau)$ and $\beta(\tau)$ defined through 11

$$
\begin{aligned}
f_{k} & =\left(2 w_{k}\right)^{-1 / 2}\left[\alpha_{k} e^{-i \int w_{k} d \tau}+\beta_{k} e^{i \int w_{k} d \tau}\right] \\
\dot{f}_{k} & =-i\left(w_{k} / 2\right)^{-1 / 2}\left[\alpha_{k} e^{-i \int w_{k} d \tau}-\beta_{k} e^{i \int w_{k} d \tau}\right] \\
w_{k}^{2} & =k^{2}+\xi R e^{2 \rho}
\end{aligned}
$$

Eq.20 reads

$$
\begin{aligned}
\dot{\alpha}_{k} & =\frac{\beta_{k} \dot{w}_{k}}{2 w_{k}} e^{2 i \int w_{k} d \tau} \\
\dot{\beta}_{k} & =\frac{\alpha_{k} \dot{w}_{k}}{2 w_{k}} e^{-2 i \int w_{k} d \tau}
\end{aligned}
$$

with the normalization condition $\left|\alpha_{k}^{2}\right|-\left|\beta_{k}\right|^{2}=1$. In what follows we will assume that $\left|\alpha_{k}\right| \simeq 1,\left|\beta_{k}\right| \ll 1$. Therefore, the equation for $\beta_{k}$ can be easily solved

$$
\beta_{k} \simeq \frac{1}{2} \int_{-\infty}^{\tau} d \tau \frac{\dot{w}_{k}}{w_{k}} e^{-2 i \int^{\tau} w_{k}\left(\tau^{\prime}\right) d \tau^{\prime}}
$$

where we assumed that the matter fields are in the in-vacuum $\left(\beta_{k}(-\infty)=0\right)$. The energy density of created particles at time $\tau$ is given by

$$
\epsilon(\tau)=2 N \int_{0}^{\infty} d k w_{k}\left|\beta_{k}(\tau)\right|^{2}
$$


From the above equations it is easy to find an upper bound for $\epsilon(\tau)$. The coefficient $\beta_{k}(\tau)$ satisfies 5

$$
\left|\beta_{k}\right| \leq \frac{1}{2} \int_{-\infty}^{\tau} d \tau \frac{\dot{w}_{k}}{w_{k}}=\frac{1}{4} \log \left|1+\frac{2 \xi \ddot{\rho}(\tau)}{k^{2}}\right|
$$

With this bound, one can estimate the integral in Eq.24 by considering the cases $k^{2} \gg$ $|\xi \ddot{\rho}(\tau)|, k^{2} \sim|\xi \ddot{\rho}(\tau)|$, and $k^{2} \gg|\xi \ddot{\rho}(\tau)|$. The result is

$$
\epsilon(\tau) \leq \alpha N|\xi \ddot{\rho}(\tau)|
$$

where $\alpha$ is a number of order one. We will set $\alpha=1$ in what follows.

From the solutions of Section II we find (see Eq.13)

$$
\ddot{\rho}=-\frac{1}{\kappa} \ddot{A}=-\frac{1}{2 \kappa} \frac{\delta^{2}}{T^{2}} \frac{A^{2}-2 A^{\prime \prime} A}{\left(A-\frac{A^{\prime 2}}{4 \kappa}\right)^{2}}
$$

The couplings $A(\phi)=e^{-\gamma \phi}$ and $\phi^{2 n}$ satisfy that $A^{2} \sim A^{\prime \prime} A$ in the weak coupling region. As a consequence,

$$
\epsilon(\tau) \leq N|\xi \ddot{\rho}(\tau)| \sim \frac{N \kappa|\xi| \delta^{2}}{T^{2}} g_{c}^{2}(\tau)
$$

where $g_{c} \sim g_{\text {eff }}$ is the $\phi$-dependent coupling constant. Therefore the energy in created particles vanishes near the singularity. This means that the analysis of the existence of singularities done in the previous Section is valid and the solutions can be trusted as long as $\epsilon(\tau) \ll \frac{m_{c r}^{2}}{T^{2}}$.

At this point one would conclude that particle creation does not help to smear the singularities. We will now argue that this is not always the case. We have chosen the coordinate $\tau$ in such a way that the singularity is at $\tau=-\infty$. Moreover, we assumed that the quantum state of the matter fields is the $i n$-vacuum. This is the reason why $\epsilon(\tau)$ vanishes on the singularity.

However, the semiclassical equations for $\xi=0$ are time-reversal invariant. As a consequence, there are solutions where the singularity is located at $\tau=+\infty$. If we choose again

\footnotetext{
${ }^{3}$ For simplicity we assume that $\frac{\dot{w}_{k}}{w_{k}}>0$ in the region of integration.
} 
$\mid 0_{\text {in }}>$ as quantum state, the singularity will survive only if, during the whole evolution, the energy in created particles does not exceed the critical value. Whether this condition is satisfied or not depends on the different parameters $(\xi, N, \delta)$ and on the coupling $A(\phi)$. In any case, we see that if there is enough particle creation the proof of the existence of singularities could be fundamentally flawed.

\section{CONCLUSIONS}

To summarize, in the generic dilaton-gravity theory considered here, when the matter content is below the threshold, the quantum effects of conformal matter fields produce weak coupling singularities as long as $A^{\prime 2} \gg \kappa A$ in the weak coupling region.

In the particular case $\lambda^{2}=0$, and when the matter content equals the critical value, the corresponding critical solution is the Milne universe. There is no solution for $m^{2}>m_{c r}^{2}$. On the other hand, when $\lambda^{2} \neq 0$, the solutions for $m^{2} \geq m_{c r}^{2}$ do exist, but they have no weak coupling singularities.

A similar critical behaviour at the onset of black hole formation has been recently discovered numerically in the S-wave sector of four dimensional general relativity, and analytically in the RST model [10]. Our 'cosmological' results suggest that, also in the black hole problem, the threshold should not depend on the coupling constants of the theory, while the scaling should depend.

Turning back to the cosmological singularities, the situation is different for non-conformal fields. In this case, if the quantum state of the matter fields is chosen to be the vacuum state near the singularity, then the singularity survives. However, for other quantum states, if the energy in created particles exceeds the critical value, the singularities could dissappear. Therefore, the semiclassical cosmological singularities seem to be generic only when conformal invariance is not broken.

Breaking of conformal invariance should also be important when analyzing the theory

beyond the semiclassical approximation. Indeed, most of the works done in $(1+1)$ dimensions 
- including this one - assume, without justification, that the spacetime metric is a classical object. This should be justified in the full quantum theory. An important ingredient in the quantum to classical transition is decoherence between macroscopic trajectories [12]. It has been shown in the context of $(3+1)$-dimensional quantum cosmology that decoherence takes place if and only if there is particle creation [13]. This result is independent of the number of dimensions. As a consequence, a theory with conformal matter fields may not have a well defined classical limit! Thus, in order to have a reasonable toy model in $(1+1)$ dimensions, conformal invariance should be broken. It would be interesting to reanalyze the black-hole puzzless in this context.

\section{ACKNOWLEDGMENTS}

We would like to thank Carmen Núñez for useful discussions. This research was supported by Universidad de Buenos Aires, Consejo Nacional de Investigaciones Científicas y Técnicas and Fundación Antorchas. 


\section{REFERENCES}

1 C.G. Callan, S.B. Giddings, J.A. Harvey and A. Strominger, Phys. Rev. D45, R1005 (1992).

2 A. Bilal and C.G. Callan Nucl. Phys. B394, 73 (1993); S.P. de Alwis, Phys. Lett. B300, $330(1993)$

3 J.G. Russo, L. Susskind and L. Thorlacius, Phys. Rev. D46, 3444 (1992); ibidem 47, $533(1993)$

$4 \quad$ F.D. Mazzitelli and J.G. Russo, Phys. Rev. D47, 4490 (1993).

$5 \quad$ Klimcik, Phys. Rev. D 48, 4693 (1993).

6 T. Piran and A. Strominger, Phys. Rev. D 48, 4729 (1993); T. Banks and M. O'Loughlin, Phys. Rev. D48, 698 (1993)

7 J.G. Russo and A.A. Tseytlin, Nucl. Phys. B382, 259 (1992).

8 N.D. Birrel and P.C.W. Davies, Quantum Fields in Curved Spaces, Cambridge Univ. Press (1982)

$9 \quad$ E.A. Milne, Nature 130, 9 (1932)

10 A. Strominger and L. Thorlacius, Phys. Rev. Lett.72, 1584 (1994); For similar results in 3+1 dimensions see M.W. Choptiuk, Phys. Rev. Lett. 70, 9 (1993)

11 Y. Zeldovich and A.A. Starobinskii, Sov. Phys. JETP 34, 1159 (1972); A.A. Starobinskii, in Quantum Gravity II, edited by M. Markov and P.C. West, Plenum, New York (1984)

12 C. Kiefer, Class. Q. Grav., 4, 1369 (1987); J.J. Halliwell, Phys. Rev. D39, 2912 (1989);

T. Padmanabham, ibidem 2924 (1989)

13 E. Calzetta and F.D. Mazzitelli, Phys. Rev. D42, 4066 (1990) 